\title{
Psychology as an Associational Science: A Methodological Viewpoint
}

\author{
Sam S. Rakover \\ Department of Psychology, University of Haifa, Haifa, Israel \\ Email: rakover@psy.haifa.ac.il
}

Received March 20 $0^{\text {th }}, 2012$; revised April 17 ${ }^{\text {th }}, 2012$; accepted April 30 ${ }^{\text {th }}, 2012$

\begin{abstract}
Unlike the sciences (physics), psychology has not developed in any of its areas (such as perception, learning, cognition) a top-theory like Newtonian theory, the theory of relativity, or quantum theory in physics. This difference is explained by a methodological discrepancy between the sciences and psychology, which centers on the measurement procedure: in psychology, measurement units similar to those in physics have not been discovered. Based on the arguments supporting this claim, a methodological distinction is made between the sciences and psychology as an associational science. It is suggested that that these two kinds of science generate two different classes of technologies. The possibility that in psychology there is a connection between the issue of measurement and the unsolved consciousness/brain problem is discussed.
\end{abstract}

Keywords: Philosophy of Psychology; Methodology; Philosophy of Science and Mind

\section{Introduction}

Since 1879, the year in which Wilhelm Wundt, whom many psychologists consider as a leading figure in founding psychology as a science, established the first laboratory for psychology in Leipzig, 132 years have passed (In view of the complexity of the history of psychology and its interpretations, I attempt to briefly describe its main changes in as factual manner as I can (e.g., Leahey, 1992, 2004)). 132 years is a considerable stretch, and calls for a comparison of the achievements of psychology with the achievements of the natural sciences. True, the latter are older (e.g., Newton published his Principia in 1687, namely 324 years ago, and Levoisier published his work on the elements of chemistry in 1789 , namely 222 years ago) (e.g., Gribbin, 2004; Leahey, 2004). These time differences notwithstanding, one salient difference between the natural sciences and psychology may be pointed out: psychology has not developed in any of its areas (such as perception, learning, cognition) a top-theory like Newtonian theory, the theory of relativity, or quantum theory in physics.

Since its founding, psychology has undergone enormous changes in its research programs. Three major changes are these: from the science of mental life, consciousness, to the science of behavior; and then to the science of cognition, information processing. At first psychology defined itself as research into conscious experience, when the main research instrument was introspection (that is, an internal observation by the individual of her consciousness for the purpose of breaking it down to its basic components). In parallel a development occurred in the research of behavior, and in 1913, in an article published in the Psychological Review, Watson heralded the behaviorist approach: research into consciousness was to be cast aside, and attention was to be concentrated on controlled observations of behavior, because while the previous approach was subjective, the behaviorist approach was objective. The late 1950s was a period of rapid development of cognitive psychology; this ap- proach maintained that cognitive processes (conscious and unconscious) had to be investigated, based on an analogy with the model of the computer as a mechanism that processes information, that is, a mechanism that represents reality in physical symbols and performs computational operations on them (e.g., Neisser, 1967; Sternberg, 2009).

Although this approach predominates still today (about 50 years on) in psychology, it too has caused unease. In the 1980s several other approaches appeared that highlight a number of aspects in psychological research that were neglected by the cognitive approach (which is essentially mechanistic): study of the personality in its entirety, importance of free will, and phenomenology (e.g., Gross, 2009). In approximately the last 20 years psychology has begun to emphasize research on neuronphysiological mechanisms in the brain and their relation to behavior and consciousness. These topics have been investigated with one of the most popular technique in neuroimaging, functional magnetic imaging (fMRI). Diener (2010) believes that "The perception seems to be that the proponents of imaging are saying that most of what went before in psychology was rather worthless, and we now finally have the effective tool to truly understand psychology in a scientific way" (p. 714). And Shimamura (2010) has proposed "... I am convinced that the 1990 s will be viewed as the beginning of the cognitive neuronscience revolution" (p. 722).

What I wish to stress in this short historical account is the changes in the development of psychology, its instability, a state having to do with the failure to develop a top-theory in any domain at all. Psychologists are liable to recoil from this depiction, to say that it is too cutting, and to pull out as counter examples several broad psychological theories, for example, Freud's psychoanalytic theory or Hull's theory of learning (e.g., Hilgard \& Bower, 1966; Marx \& Cronan-Hillix, 1987). True, these theories won great popularity in their day, but within about two decades it transpired that they had failed empirically 
and theoretically. Today it is hard to find researchers who believe in them (The great influence of psychoanalysis on literature and on everyday discourse is a different story altogether, because this influence is in no way an empirical test of psychoanalytic theory by the methodology of science.). Even learning theory that Pavlov developed in his day has been refuted-although his experiments are still today the cornerstone in the field of animal learning (see Kimble, 1961). Several recent attempts to propose a general theory, such as Newell's (1992) SOAR, were developed on the basis of the AI approach to cognition. Although SOAR has made significant contributions to understanding cognition, it has received much criticism and has aroused controversies (see, e.g., Cooper \& Shallice, 1995; Garcia-Marques \& Ferreira, 2011; Lewis, 2001). Recently, De Houwer, Fiedler, \& Moors (2011) have suggested that the era of gathering empirical findings has ended and the time has come to focus on developing theoretical explanations.

It is important to emphasis that even cognitive psychology has not developed a top-theory. In 1973 Newell wrote a famous article following a conference on processing visual information, in which he expressed pessimistic views on the condition of psychology. He summarized the presentations and concluded that cognitive psychology could be regarded as a collection of interesting phenomena that were not explained by a uniform cognitive theory. Each phenomenon was explained by local hypotheses, namely those linked directly to the given phenomenon, where these ultimately came down to two contrasting hypotheses, for example, memory based on one mechanism as opposed to two mechanisms. This state of affairs, Newell suggested, was likely to continue for many years. And sure enough, as it turns out, it continues to characterize cognitive psychology to this day: psychological research is fractionated (e.g., Garcia-Marques \& Ferreira, 2011; Meiser, 2011).

The question is how may this difference-the lack of toptheory in psychology-between psychology and the natural sciences be explained? The answer, I believe, lies in the wide methodological difference between the sciences and psychology, which centers on the procedure of measurement. So first I shall consider this difference, which separates two kinds of science: one that characterizes physics, chemistry, and the other fields of the natural sciences, and the other a science that I call "associational science", which is characteristic of psychology. Then I shall consider the implications of this science for technology. At the end I briefly speculate on the possible connection between this difference and the mind/body (consciousness/brain) problem.

\section{The Measurement Problem: In Psychology Measurement Units Similar to Those in Physics Have Not Been Discovered}

To fathom the difference between these two kinds of science I have first to briefly outline a theory in the sciences on whose structure a psychological theory has been developed (e.g., Rakover, 1990, 2007; Suppe, 1977, 1979). One may roughly sketch the structure of a theory in physics (for simplicity I will refer in the present paper to classical physics) as based on two levels of analysis, the theoretical and the observational, where the theoretical level represents and explains certain aspects of the observational level, the phenomenon under study. For example, Galileo's law accounts for a free-falling body by appealing to the force of gravity and by representing time and distance of the falling body (see below). Similarly, a psychological theory may be seen as based on these two levels of analysis:

Theoretical level: $\{$ Stimuli \& Processes $\} \rightarrow$ Behavior Observational level: STIMULI $\rightarrow$ INDIVIDUAL $\rightarrow$ BEHAVIOR

On the theoretical level we try to predict the INDIVIDUAL's BEHAVIOR (Behavior) by means of representation of the STIMULI (Stimuli) and certain hypothesized Processes (cognitive, neurophysiological), that is, Behavior $=\mathrm{f}$ (Stimuli, Processes). The theoretical level, therefore, represents certain aspects of the observational level and attempts to reflect the processes taking place in the individual, which are responsible for her behavior in a given state of stimuli. (Note that by this formulation of the structure of a theory as based on two levels of analysis - theoretical and observational, it is not obligatory to distinguish sharply theoretical from observational concepts, a distinction that has been criticized severely. Despite this criticism, in psychology this distinction is customarily made between theoretical concepts such as information, processing information, perception, and short-term memory, and observational concepts such as reaction-time, response strength, and correct response, see Rakover, 1990.)

The major difference between psychology and the sciences resides in the attempts to connect the concepts on the theoreticcal level to the concepts on the observational level (to bridge the theory-observation gap). The difference is expressed in an important measurement property. While in physics the bridge over the theory-observation gap is based on the use of real measurement units (e.g., a ruler to measure distance and a balance to measure weight), in psychology the bridge is based on the use of hypothetical measurement units that are indexed by one's responses (e.g., a just noticeable difference in psychophysics is indexed by one's decisional responses). So while in physics one develops a theory that uses theoretical concepts connected to observations through the use of real measurement units, in psychology theoretical concepts are connected to observations (behavior) through the assumption of hypothetical measurement units. This measurement property (real/hypothetical) is associated with several other interesting measurement properties, which are discussed below. To clarify the difference, I exemplify first the way physics measures its basic concepts that appear in its laws and theories; then I will consider psychology.

Let us look as an example at Galileo's law, the law of freefalling bodies: $S=1 / 2 \mathrm{GT}^{2}$, where $\mathrm{S}$ stands for distance, $\mathrm{T}$ for time, and $\mathrm{G}$ acceleration of the body due to the force of gravity. The important point I wish to stress in this example is the way in which concepts are measured, that is, the empirical measurement: $\mathrm{S}$ distance and $\mathrm{T}$ time $(\mathrm{G}$ is a theoretical concept that cannot be measured in the same way as distance and time). ${ }^{3}$ Without entering into the ongoing debate about the various theoretical approaches to such basic terms as property, number, and measurement, here I describe measurement as a process in which the relation between a certain quantitative property of a given object and a unit of measurement of this property is revealed empirically (e.g., Coombs, Dawes, \& Tversky, 1970; Hand, 2004; Michell, 1999). For example, if we have a straight stick of length $A$, and we find that another stick of length $M$ (which we arbitrarily determine as our unit of measurement, e.g., the meter), goes into A ten times from end to end, we have discovered that length $\mathrm{A}$ is ten lengths $\mathrm{M}$ (i.e., $\mathrm{A} / \mathrm{M}=10$ ). $\mathrm{Al}$ - 
though what we have found here seems trivial at a glance, this procedure carries huge significance, on which physics rests. Why?

The essential point in this measuring is that scientists found an empirical operation (counting how many times $\mathrm{M}$ goes into the length of the measured object), which upholds mathematical properties that define the world of numbers on which the mathematical signs in physical theory are based. To illustrate this, let us look at the following two properties: transitivity and additivity. The transitive relation states, for example, that if (20 $>10)$ and $(10>5)$, then $(20>5)$; and the additive relation proposes that $(5+15)=20$. These relations exist in the group of sticks A, B, C:

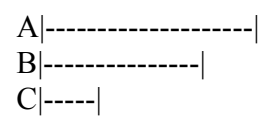

As a first step, we define a natural and arbitrary unit of measurement of length by means of the section (-); as a second step we count how many times this unit goes into A (20 times), into B (15 times), and into C (5 times); and as a third step, we see that indeed the lengths of the three sticks uphold the transitive relation, because $\mathrm{A}$ is greater than $\mathrm{B}, \mathrm{B}$ is greater than $\mathrm{C}$, and $\mathrm{A}$ is greater than $\mathrm{C}$; and also the additive relation, because $\mathrm{A}=\mathrm{B}$ $+\mathrm{C}(20=15+5)$. In other words, measuring the length allows scaling the group of sticks not only on the ordinal scale (whose values are arranged according to the relation $\mathrm{X}$ is greater than $\mathrm{Y}$ without knowing by how much, as, for example, in military ranks), but also on the interval scale (whose values are arranged according to the relation $\mathrm{X}$ is greater than $\mathrm{Y}$ by a certain quantity, for example, A is longer than B by 5 units of length).

Measurement of length of an object by means of a natural, real and arbitrary unit of measurement maintains all the mathematical properties of numbers, so it transpires that what we say by means of numbers will also be said by means of the lengths of the sticks. And this, to my mind, is the strongest tie that may be made between theory and observation. The same may be said of several quantitative properties of this kind, for example, weight and time. (Measurement of weights is based on the use of scales, and measurement of time is based on the use of a periodic phenomenon, such as the earth revolving around the sun. Physics utilizes other quantitative measures that I shall not deal with, because the measurement theory that underlies them is not simple, and is not presentable easily and intuitively like measurement of length.)

And now let us pass on to psychology: in psychology, is the connection between theory and observation based on the procedures outlined above? It is not. Psychologists connect concepts to observations by utilizing an "operational definition," which holds that the meaning of a concept is obtained by detailing the processes of observation. For example, learning is defined by means of counting the correctly solved problems; aggression is defined by the number of words or motor movements deemed threatening in a certain culture; fear is defined by distance of flight; time is defined by subjective estimate of the physical time lapse between two events; and the cognitive effort required to solve a given problem is defined as the length of time-latency - it takes to solve this problem. Do these examples substantiate that in psychology the theory-observation relation is as close as in physics? In my view they do not, principally because of the above-mentioned difference: for the physical dimensions, real units of measurement have been dis- covered (e.g., of length, weight, time); but for the psychological dimensions (e.g., sensation, perception, memory, i.e., the individual's inner world) no such units have been revealed. Three important measurement properties are associated with this difference.

Objectivity: like physicists, who attribute numbers to physiccal properties, for example, the length of this rod is five meters (i.e., the unit of a meter goes five times into the rod from end to end), psychologists attribute numbers to psychological properties, for example, on a scale that measures interest from one to ten, David's lecture is graded eight; the number of David's correct answers in the task of face recognition is seventy out of a hundred; and so on. Is assigning numbers in psychology endowed with the same measuring properties as it is in physics? Again, it is not.

In physics, scientists ascribe numbers to physical properties existing in the world, outside the human cognitive system; but in psychology numbers are ascribed not to behavioral properties existing in the world outside the human cognitive system, but to stimuli as the human perceives them. As an example, we may look at the famous Müller-Lyer illusion:

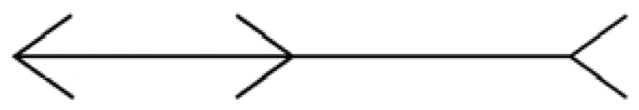

The physicist finds that the length of the right-hand line is identical to that of the left-hand line, but the participant in the psychology experiment ascribes a smaller numerical value to the left-hand line than to the right-hand one. The reason is this: while the physicist assigns numbers to physical properties located outside the cognitive system, that is, he finds that the unit of measurement of length goes precisely the same number of times into the right-hand and into the left-hand line, the person in the psychology experiment responds to the right-hand and the left-hand stimuli according to the information processing that is taking place in her perceptual system and finds signifycant expression in her consciousness: the left-hand line is shorter than the right-hand one.

A researcher who believes that measurement in psychology equals the measurement in physics may propose that the size of this illusion can be measured by the following procedure: move the right-hand line to the left until it looks the same as the left-hand line. The difference between the subjectively adjusted length and the objective length is considered an index reflecting the size of the illusion. However, the index is no more than an expression of the information processing taking place in one's perceptual system. In fact, this procedure results in what is called the "point of subjective equality", which is different from the "point of objective equality" measured by a ruler.

The logic behind this comment is relevant to other indexes used by psychologists, such as reaction time (latency) and percent of correct responses. As an example, let us consider recognition of faces, which is researched by employing the popular procedure of yes/no recognition. In the study stage the participants view 20 different faces, and in the test stage they are shown 40 faces ( 20 old and 20 new) and are asked to decide for each face whether it is old or new. Face recognition is indexed by percent correct recognition (100x number of correct recognitions/40). This index is based on the participant's old/new response resulting from a certain hypothetical cognitive mechanism that processes facial information. Face recognition is not based on the physical visual form of the face per se. If it were, one would predict that recognition of an upright face equaled 
recognition of an inverted face - a prediction that contradicts the face inversion effect (where recognition of inverted faces is much lower than that of upright faces (e.g., Rakover, 2002a). Given this, percent correct cannot be viewed as an objective index in the sense described by the measurement of length. As a behavioral index of recognition, percent correct can be biased by several factors, for example, motivation. These biases can be reduced by employing the Signal Detection Theory, which proposes an improved index for recognition (the parametric d' or the non-parametric A') that is based on the participant's responses classified as hits and false-alarms (e.g., Macmillan \& Creelman, 2005). Still, the connection between the improved index and the theoretical cognitive mechanism is hypothetical and therefore measurement in this case is different from that conducted in physics.

Separability: in physics, measurement of length, done with a real measuring device (ruler, yardstick), is separable from the phenomenon measured, whereas in psychology the measuring is a part of the psychological phenomenon, of the individual's response to the state of stimulus, and they are inseparable. We may illustrate this difference by a comparison of two experiments: one in physics, the other in psychology.

Let us test Galileo's law. A simple way is to measure the distance that a body in free fall travels in the course of one second, two seconds, and so on, and to compare these distances with the predictions of the distances derived from this law. The method of conducting this experiment is straightforward, for example, by photographing an iron ball falling freely with a camera that is activated each second. So now, all we have to do is measure with a ruler the distances the ball has traveled in the photos, match the size of the photo to reality, and make the required comparisons. The point I want to emphasize with this example is that the use of a device (a ruler) to measure the distance is separate from the behavior of an iron ball in a state of free fall. Does a distinction of this kind exist in psychology? It does not.

To check this answer we shall examine a standard experiment in psychology to measure the difference threshold [known also as "just noticeable difference" (JND)], which may be considered as connected to a unit of measurement of the psychological dimension (sensation and perception) (e.g., Baird \& Noma, 1978; Gescheider, 1997; Stevens, 1975). The threshold was discovered in experiments testing this question: given a certain stimulus, what is the minimal change in this stimulus for us to sense a change? For example, if a room is lit at certain intensity, what is the minimal change in the light for the individual to feel a change? Weber was the first researcher to find that the difference threshold increased in fixed relation to the increase in intensity of the physical stimulus for a given sensory dimension (an empirical generalization called Weber's law). For example, according to his law a stimulus of intensity 11 has to be presented for sensing a change in the stimulus of intensity 10 , but a stimulus of intensity 1100 has to be presented for sensing a change in the stimulus intensity of 1000 . And another example: if we have 10 dollars, the addition of 1 dollar is significant; but will the addition of 1 dollar have the same significance if we have in our possession 10,000 dollars? Given Weber's law, Fechner assumed that the changes in the difference threshold match particular changes in the sensory dimension, i.e., increase in the physical dimension matches increase in the number of sensory measurement units of equal size. This theoretical assumption about the sensory measurement unit led to the development of Fechner's law, stating that sensation equals the logarithm of the intensity of the physical stimulus [that is, the geometric change in the physical dimension (large change) is translated into arithmetical change in the psychological dimension (small change)].

However, the concept of a sensory measurement unit is no more than a hypothesis, whose methodological status is not parallel to the real unit of measurement of length (the meter) and its use which is separable from the investigated phenomenon. This hypothesis obtains its strength from empirical findings that accord with the predictions derived from Fechner's law. But not only do some findings not support this law, alternative theories and laws have even been proposed. One of these is Stevens' power law, developed on the basis of a hypothesis contrary to Fechner's: not only the physical dimension behaves according to Weber's law, so does the psychological (sensation).

Conscious experience: in most cases in psychology participants' responses to stimuli are not a kind of purely motor movements, automatic reflexes, but are responses carrying meaning and consciousness. I raise my hand not as a pointless motor movement but to say hello. Our responses, our actions, and our deeds are replete with intentions, wishes, desires, feeling of awareness - that is, with conscious experiences.

Still, for the conscious experience no unit of measurement has as yet been found as it has for length. For example, one cannot take the conscious experience of a taste of lemon (not the physical stimulus, the lemon juice, which can be measured with great precision), define for it a measurement unit of the sensation of lemon taste (the meter of the lemon, $\mathrm{M}_{\mathrm{L}}$ ) and use it to measure the sensation of lemon taste $\mathrm{L}$, so that we would obtain, for example, $\mathrm{L} / \mathrm{M}_{\mathrm{L}}=10$, that is, the sensation of lemon taste $\mathrm{L}$ is ten times $\mathrm{M}_{\mathrm{L}}$ (ten units of sensation of lemon taste). It is not possible to take the emotion of love, define a measurement unit of love (the meter of love, $\mathrm{M}_{\text {love }}$ ), and say that Jacob loves Rachel ten and half units of love more than he loves Leah. By this example I do not mean to suggest that one cannot say that Jacob loves Rachel much more than Leah, only that a measurement unit of love $\left(\mathrm{M}_{\text {love }}\right)$ has not been discovered like the natural unit of length (the meter) and in this respect it is not possible to measure Jacob's love for Leah, and say that it is 10 $\mathrm{M}_{\text {love }}$ while his love for Rachel is $20.5 \mathrm{M}_{\text {love }}$.

Here a qualification is needed. It may be said that use of a measuring unit (e.g., the unit of a meter) is a sufficient condition for measurements to uphold properties of an interval scale (like the scale of natural numbers), but this is not a necessary condition. The reason is that although for psychological properties natural measurement units like the length unit have not yet been discovered, findings have been obtained that show that in certain cases a group of responses to several stimuli approaches the properties characteristic of an interval scale. For example, in the tone-bisection task a participant hear two tones, 1) high loudness; and 2) low loudness, and is asked to produce subjectively a tone whose loudness is halfway between these two tones. Based on this task, a mathematical model has been developed that generates an interval scale on which it was possible to scale the tones produced by the participant, i.e., a good match is revealed between the predictions derived from the model and the participant's behavior (e.g., Coombs, Dawes, \& Tversky, 1970).

Unit equivalency: the situation where psychology is not endowed with measurement units as in physics is expressed in its inability to fulfill the requirement I call "unit equivalency" 
(based on dimensional analysis) (e.g., Rakover, 1997, 2002b). According to unit equivalency, the combination of measurement units on one side of the law's or the theory's equation must be identical to the combination of the measurement units on the other side of the equation. To explain this requirement, let us look once more at Galileo's law, that of free fall of bodies: $\mathrm{S}=1 / 2 \mathrm{GT}^{2}$. Now, since $\mathrm{S}$ is measured by the meter unit, the expression $\mathrm{GT}^{2}$ must also be measured by the meter unit. And indeed, a simple calculation shows that it is: meter $=($ meter/ time $^{2}$ ) time ${ }^{2}$.

Does a psychological theory meet the requirement of unit equivalency? It does not. Let us look for example at the overall structure of theory in psychology: Behavior $=\mathrm{f}$ (Stimuli, Neurophysiological processes, Cognitive processes). Now we ask if the measurement units of the behavior are identical to the measurement units of the stimulus, the neurophysiology, and the cognitive processes. They are not. For example, the number of correct responses is not identical to the physical units of the brain processes (differences of electrical potential), to the units with which the stimulus is measured (loudness of the noise), or to the measurement units of cognitive processes (information processing).

Yet here too a qualification is needed. For simplicity, I shall look at the following fictitious and amusing example. Assume that an association has been found between shirt size and intelligence size (IQ): up to a certain age (the teens), as the size of the shirt grows, so does IQ. Now let's assume, again for simplicity and frivolity, that the researchers have expressed this connection in a "law", which I shall call "intelligence-shirt": IQ $=\mathrm{aS}$, where $\mathrm{S}$ signifies the shirt's size and IQ signifies intelligence. Clearly, this law does not meet the requirement of unit equivalency, because the right side of the equation is measured in centimeters and the left side in number of correct answers in an intelligence test. But if one interprets the coefficient a as expressing the intelligence/centimeter relation she may say that even this law fulfills the present requirement (the equation has intelligence on either side). The problem is that this interpretation is ad hoc, namely it is a contrived solution tailored-made especially for this particular coefficient, and in no way parallels the interpretations of the coefficients common in the sciences, which are stable and their estimates do not change in different conditions, i.e., they are general. For example, if another connection is discovered between Normativeness (behavior in keeping with social norms) and intelligence, $\mathrm{IQ}=\mathrm{bN}$, it will be possible to interpret the coefficient $b$ in the new "intelligencenormativeness" law as expressing the intelligence/normativeness relation, and so on, up to an infinity of "laws" of this kind. An additional problem to do with the intelligence-shirt law is that the intelligence scores cannot be arranged on an interval scale (hence their values are not addable or multipliable) whereas centimeters can be arranged. Hence, from a mathematical viewpoint it makes no sense to divide the intelligence score by the shirt's size as required by coefficient a (intelligence/centimeter).

In many cases an odd situation seems to be created in which psychology plays the math game correctly without reflecting psychology itself. Consider the following intuitive examples. Assuming that Einstein's intelligence level was very high (IQ = $150)$, is it possible to argue that his intelligence level was equal to the total intelligence level of three imbeciles each of whom had IQ $=50$ ? And another example: it makes sense to say that if the height of the Rubinstein Tower is 100 meters, and the dis- tance between town A and town B is 10 kilometers, then the distance between these two towns is 100 Rubinstein Towers. By contrast, it is hard to say that if success in a literature test is $20 \%$ and success in a math test is $80 \%$, then the success in math is 4 successes in literature.

Measurement properties: I sum up the present discussion by suggesting four major measurement properties by whose means the measurement units in physics (e.g., length, weight, time) and their use may be characterized; these properties have no parallel in measurements conducted in psychology:

1) Real unit: real and not hypothetical units are used and are selected arbitrarily according to convenience of operation;

2) Objectivity: The units are not affected by factors operating on the phenomenon under study or by the measuring process itself (if this property did not hold, objective measurement would not be obtained either);

3) Separability: The units and their use by means of a measuring device (e.g., ruler, meter, weight) are separate from the phenomenon being measured;

4) Mathematical representation: Measurement by means of real units (e.g., length, weight, time) are represented by numbers (on interval or ratio scales), so that what is obtained in numbers is obtained precisely in measurements in physics, and the requirement of unit equivalency is fulfilled.

As can be seen from this list of measurement properties, the methodological difference between physics and psychology is much more than the mere declaration that IQ scores are not scaled on an interval scale-a statement that students in psychology learn in their first year. Since psychology does not reveal these properties, one may propose that while the theory-observation gap is tightly bridged in physics, it is loosely bridged in psychology.

\section{Science and Associational Science}

This difference between physics and psychology in measurement procedure and units brings me to the methodological distinction between science (physics, chemistry, etc.) and an associational science (psychology). According to this proposal, not everything that can be realized methodologically in science can be realized in psychology, but everything that can be realized in psychology can be realized in science. First I shall try to describe what can be realized methodologically in psychology as an associational science.

In most research areas of associational science it is possible to use methods such as conducting observations, proposing explanations, constructing hypotheses and theories and testing them empirically, constructing research and experimental designs, analyzing results, and writing a scientific reports. However, contrary to science, the above measurement properties do not characterize measurement in psychology: the measurement unit is hypothetical, is not objective in the sense that it is affected by the factors affecting behavior under study, is not separable from behavior, and does not fulfill all the mathematical requirements of numbers.

Still, the research methods that can be utilized in psychology allow an interesting and important accumulation of knowledge. One may examine two kinds of association: stimulus and response (response and stimulus), and response of one kind and response of another kind. These associations can be investigated by means of various research designs and statistical analysis. For example, it is possible to investigate: the stimulus that ef- 
fects the individual's response as compared with other stimuli; the situation in which a behavior will be deemed rational (e.g., by maximization of profits); the nature of the connection between two responses such as that between neurophysiological indexes (e.g., pulse, perspiration, and neuroimaging) and the individual's responses in tests of perception, learning and memory. In all these instances, and their like, hypotheses may be developed that explain the empirical link found in certain conditions. In some special cases the link may be characterized as a stable phenomenon which psychologists tend to take as an empirical law (e.g., Weber's, Fechner's, and Stevens' laws). (However, Uttal, 2008, argued that these laws and others like them may be artifacts, since their development involves the process of averaging).

In science the methods practiced in associational science can be operated, and it is also possible to operate fully the methods based on the above measurement properties. These methods become possible, as stated, because in the sciences (e.g., physics, chemistry) natural and real measurement units have been discovered. By means of the measurements of length, weight, time (and other measurement units) physics has succeeded in developing complex concepts such as speed, acceleration, work, and energy, based on combinations of these units of measurement. The development of these concepts imparted to researchers the ability to develop equations that represent laws of conservation and conversion of energy, for example, the law of conservation of energy, converting mechanical energy into heat, converting magnetism into electricity and the reverse, turning mass into energy, and explaining chemical properties by chemical equations, where on either side the overall weight and number of atoms are preserved. These achievements have made it possible to develop general theories that explain a large collection of empirical phenomena.

In psychology no such theoretical-empirical development has occurred, because measurement units have not been discovered and full use of the above measurement properties is impossible. This situation is expressed in two important problems: validity and multiplicity of dimensions.

To illustrate the validity problem we shall look at the term "cognitive effort", which is measured by latency (time of response from the moment of presentation of the stimulus). Does latency indeed measure solely the cognitive effort, in a way similar to the theory-observation connection in physics? It does not. Measuring the length of an object with a standard ruler centers on this alone, and does not measure the color and kind of material of which the object is made. Latency, by contrast, is influenced by a large number of factors and various cognitive processes, for example, tiredness, excitement, and interest, which have no direct connection to the cognitive effort needed to solve a problem. Furthermore, latency does not mirror only the process connected to this effort, because it is not known which cognitive processes go into action when the individual tries to solve a problem. For example, does the individual need more information located in her memory, and what is the speed of retrieval of this information? Do these processes operate one after the other or in parallel? A similar criticism may be leveled against basic concepts in psychology: information and information processing. In physics and the computer sciences these concepts are defined as exact, but in psychology they form a kind of catchall drawer in which one may place whatever there is to be said about knowledge and the various ways in which the brain treats knowledge, such as content, meanings, associa- tions, ways of coding, and hypothetical storage and retrieval (e.g., Pachella, 1974; Palmer \& Kimchi, 1986).

The concepts of psychology are multi-dimensional, and interpretable from different and varied viewpoints, but the concepts of physics (length, weight, time) are one-dimensional. Moreover, it is very hard to break down psychological concepts into their one-dimensional components (try, for example, to break down the concepts of love and envy, perception, memory, or ego), but in physics the complex (multi-dimensional) concepts are composed of one-dimensional concepts. For example, speed is based on the relation between distance and time; acceleration is based on the relation between distance and time squared; and kinetic energy is based on the relation between weight and speed squared. Furthermore, in psychology, because the concepts are multi-dimensional, in many cases the transitive relation is also broken: for example, our friend David believes that Ruth is lovelier than Dana because of her green eyes; Dana is better looking than Alisa because of her raven hair; but he insists that actually Alisa is more beautiful than Ruth because of her long fingers.

The conclusions that arise from the present discussion on the situation of psychology are the following: its concepts are vague and are loosely connected to observation - the concepts are not anchored to measurements as in the sciences. As a result, it is not easy to develop in psychology a top-theory under which it would be possible to organize coherently various hypotheses, and to explain a large number of findings; this situation is instable, and allows dramatic changes in the conception of psychology as a scientific discipline and in its research directions - two conclusions in keeping with what was described at the beginning of the article.

Implications for technology: The above discussion carries an interesting implication for the kind of technology that psychology is able to develop. If we take technology as a research field concerned with applying scientific knowledge to realize practical goals, I propose the following two intuitive notions. First, if one accepts that psychology is characterized as an associational science, it will not be able to develop technologies based on a higher level of knowledge than that about associations between stimulus and response, between response and outcome, and between responses. Second, psychology has not developed a technology like that developed by the natural sciences, namely a far-reaching technology that has transformed the face of the entire world. That is, psychology has developed a secondary technology.

First, I briefly discuss the first point-associational science. Psychological scientific knowledge has been applied to diverse areas of behaviors: clinical psychology, education, human factors, sport, communication and advertising, industrial/oganizational psychology, eyewitness testimony-where each area is divided into many sub-areas of application. Although this range of technological applications is very impressive, it is based on associational knowledge. For example, hearing impairment is tested by psychophysical methods, in which the participant responds to a series of stimuli by means of which it is possible to obtain information about the area in which a decline in the participant's hearing has taken place; analysis of intelligence tests facilitates giving a practical recommendation, for example, an examinee who has passed the test with a high score has a better chance of succeeding at other tasks sharing a common denominator with this test; but an examinee who failed the test has low chances of succeeding in those tasks. And another ex- 
ample: police technology of identifying a suspect by use of a photo album of criminals or by a facial composite is based essentially on empirical findings that face recognition is one of the best methods for probing memory. It is easy to see that in these examples the knowledge applied for practical goals is based on the participant's responses to a certain group of stimuli, i.e., the applied information is based on the methodology of an associational science.

Contrary to associational technology, science is able to offer technologies based on laws of conservation and conversion of energy. As the number of examples of technology of this kind is very great and calls for in-depth understanding of sciences, I shall describe in brief a simple example familiar to all. The flashlight is an implement based on a chemical process (in the battery) producing electricity (recorded on the battery is how much current it supplies per hour), which passes through a resistance (the filament in the bulb) and is converted into heat and light. All these conversions are subject to calculations and precise measurement, so that with knowledge of the chemical structure of the battery it is possible to calculate the intensity of the electric current, and with additional information of the resistance, it is possible to calculate the intensity of the light supplied by the flashlight.

Now I discuss the second point-influence. The impacts of the two groups of technologies (science vs. associational science) may be evaluated by using several comparisons. The first comparison is based on an if-thought game. What would be the effects on our life, if various technologies (which we use routinely everyday) had not been developed by the sciences as against psychology? The answer is clear: the nonexistence of the sciences' technologies would impact our lives much more than the nonexistence of psychological technologies.

The second comparison concerns the realization of the same goals. For example, the degree of efficiency of generating a facial composite falls far short of the efficiency of identifying the suspect by means of DNA. The use of medications for treating of various severe mental disorders (e.g., schizophrenia) is more efficient than a psychological therapy.

Finally, many applied developments in psychology depend on the technological developments in the sciences. For example, the technology of human-computer interaction developed after the computer was invented and mass produced. Many psychologists use in their research sophisticated instruments, such as computers, polygraphs (perspiration, breathing rate, and pulse), recording of brain cell activity, and neuroimaging. These technologies were not developed in psychology but in the sciences. Psychologists have used them to achieve their goals, so as to present stimuli in a controlled manner and to measure certain internal responses (e.g., neurophysiological activity in the brain). These measures are of great importance for advancing cognitive-neurological knowledge (e.g., to support/refute hypotheses about cognitive and neurophysiological mechanisms). However, they are not a kind of a mirror of the individual's conscious experiences. Mental states are not identical to neurophysiological states. One may not propose that fMRI photographs provide us with pictures of thoughts and emotions, since they only measure physical changes taking place in the brain based on changes in the blood flow (see Miller, 2010). So in these cases too, psychology is not capable of stepping beyond the associational level between stimulus and response, response outcome or between response and response.

\section{A Possible Reason for the Measurement Problem in Psychology}

The discussion so far has concentrated on the description of the measurement problem in psychology as an associational science: measurement units in psychology have not been discovered as they have in physics. As mentioned above, measurement units for the conscious sensation of the lemon taste or for that wonderful feeling of love have yet not been found. How may this problem be explained? For an answer, I speculate that the measurement problem in psychology is linked to the mind/body (consciousness/brain) problem. The main question in this regard is this: what are the implications of solving the mind/body problem for solving the measurement problem in psychology? (Here I consider two important theories of mind/ body: identity theory and reduction, e.g., Kim, 1996; Polger, 2004; Rakover, 1990, 2007). To answer the question I will examine all four possible relations between the mind/body problem (solved-unsolved) and the measurement problem in psychology (discovered-undiscovered measurement units): 1) solved/discovered; 2) solved/undiscovered; 3) unsolved/discovered; and 4) unsolved/undiscovered. As we shall see, the most feasible relation is 4) unsolved/undiscovered.

Let us start with the suggestion that the mind/body problem can be solved by identifying mental states (e.g., pain, hunger) with neurophysiological states as suggested by identity theory. If this suggestion holds, then mental states could be measured like neurophysiological states, since they are identical. In this case the measurement units used in the sciences will be used in the psychological realm and there will be no methodological difference between the sciences and psychology. A similar argument can be made for reduction. Furthermore, if indeed it were possible to reduce a psychological theory to a neurophysiological theory, one could forgo psychological concepts altogether since behavior would be explained through the theories prevailing in the sciences. McCauley \& Bechtel (2001) write "... if psychological theories map neatly onto neuroscientific theories along the lines that classical model specifies, then our commitments to psychological states and events are, at very least, dispensable in principle." (p. 739). This line of reasoning suggests that while relation 1) solved/discovered is possible, relation 2) solved/undiscovered is not (since a mental state is or reduces to a neurophysiological state).

But this is not how matters stand at present: the mind/body problem, the consciousness/brain problem, has not been solved, and as a result relation 1) solved/discovered does not seem likely (e.g., Ludwig, 2003). There are two major obstacles in the way of achieving a successful reduction of psychology to neurophysiology: multiple realizations and consciousness (see, e.g., Polger, 2004; McCauley, 2007; Rakover, 2007). According to the former, psychological states can be realized by multiple neurophysiological states (e.g., fear is realized in different animals). Hence, it is hard to propose a psycho-physiological bridging law that will connect a psychological state with a neurophysiological state (since the relation between these two is one to many), and as a result reduction of psychology to neurophysiology is blocked.

The latter problem (consciousness) comes down to the inability to understand, to explain, how neurophysiological activity in the brain (for which science is proposing ever better explanations) gives rise to the conscious experience of each and everyone, a subjective experience that only the individual is 
able to observe and that makes her a unique personality (e.g., Bayne, 2009; Chalmers, 1996; Cosmelli, Lachaux, \& Thompson, 2007; Koudier, 2009; Kriegel, 2007; Levine, 1983; Rakover, 2007; Rowlands, 2009). It is interesting to note in this context that Fechner in his day thought that the law he discovered (i.e., that sensation is a logarithmic function of physical stimulus) was a solution to the mind/body problem. He believed in the double-aspect approach to this problem, that is, that physical phenomena and psychological phenomena are nothing more than two aspects, views, of the same entity, and that his law expressed the connection between them-an approach for which supporters are hard to find today (e.g., Gescheider, 1997; Rakover, 1990).

Now let us consider the suggestion that the mind/body problem cannot be solved. Relation 3) unsolved/discovered raises an interesting possibility: while the mind/body problem is not solved, measurement units which are functionally similar but not identical to those used in the sciences are discovered in psychology. If this held, one would be able to develop complex measurement indexes, psycho-neurophysiological compound indexes, which would combine measurements in psychology and in neurophysiology. This development may allow one to develop a unified theory which would handle mind and body under one theoretical umbrella (e.g., the theory would fulfill the requirement for unit equivalency). However, this is not how matters stand at present: as argued above, measurement units similar to those used in the sciences have not been discovered in psychology. Hence, it seems that the only relation that fits the present state in psychology is relation 4) unsolved/undiscovered.

In sum, one may propose that the measurement problem is an expression of the mind/body problem: were the mind/body problem solved, the measurement problem in psychology would also be solved; but the mind/body problem has not been solved and measurement units similar to those used in the sciences have not been discovered.

\section{Conclusion}

In view of the above, what answer may be given to the old and difficult question: is psychology a scientific discipline? The answer is yes, psychology is a science, but a limited science in methodological terms; an associational science, a science whose methodological limitation seems to be connected to the problem of measurement.

Can this conclusion be generalized to other areas in the social sciences such as social psychology and sociology? My answer is yes. The measurement problem cuts across various areas of research in the social sciences. As long as researchers in these areas measure human behavior by employing behavioral indexes such as reaction time, correct responses, and evaluations, whether collected in experiments or by questionnaires, these indexes are susceptible to the critique developed here (the indexes lack the measurement properties discussed above). Hence, one may suggest that these areas of research exhibit characteristics of an associational science.

\section{Notes}

1) The difference between psychology and the natural sciences may be explained by other factors, such as the increase in the number of empirical phenomena which have lives of their own (e.g., Garcia-Marques \& Ferreira, 2011). Even if one accepts that the number of important experiments in psychology is increasing, this by itself cannot account for the difference, since it seems reasonable to suggest that the number of important experiments is increasing in the sciences also. For other explanations of the differences between psychology and the sciences suggested through the history of psychology see Leahey, 2004. The major thesis of the paper is that this difference can be explained by showing that psychology lacks particular important methodological properties connected to the procedure of measurement.

2) Lewin (1936) proposed the relation Behavior $=\mathrm{f}$ (Person, Environment) to point out that behavior at a given moment depends on the person and the situation.

3) G may be conceived as a theoretical term that is connected to certain observational as well as other theoretical terms. Similarly, psychologists have attempted to define theoretical terms such as fear by connecting them to stimulus, response, and other theoretical terms. However, while different measurements of $\mathrm{G}$ in different situations resulted in the same value, measurements of fear in different situations resulted in different behavioral indexes, which aroused contradictory theoretical interpretations (e.g., Rakover, 1975, 1980; Schwartz, 1989). Furthermore, the attempt to grasp consciousness as a theoretical term (that cannot be observed directly and is defined in terms of its relations with stimuli, responses and other theoretical terms) is problematic. This is because consciousness is a special observational term: everyone can observe directly her own consciousness, but not that of another.

4) In a way similar to what is stated in note 1 , here too one may propose different explanations to the measurement problem in psychology. One may suggest that this problem can be approached by applying the intriguing distinction made by Hacking (1995) between "human kinds" (abused children and unemployed people) and "natural kinds" (gold, particles and cats). The former (but not the latter) are characterized by the "looping effects": feedbacks that change human kinds when people become aware of being categorized and evaluated. If this distinction holds, it is possible to suggest that the measurement process affects the behavior under study and measurement itself (see also Richards, 2010). Hence, the discovery of measurement units similar to those discovered in physics may be hindered by the looping effects. (For criticisms of Hacking's approach, see Cooper, 2004.)

5) In a review chapter, McCauley (2007) criticized the multiple realization and the consciousness arguments against reduction. Although the discussion of these topics is beyond the scope of the present paper, it should be mentioned that the criticisms do not seem to deliver these arguments the coup de grace. For example, with regard to the consciousness argument, he suggested the Heuristic Identity Theory (HIT) (developed by him and Bechtel) as a methodological tool to enhance research in the interaction between psychology and neurophysiology, and not as an account of how consciousness emerges from the neurophysiology of the brain.

\section{Acknowledgements}

I express heartfelt thanks to Shmuel Ahitov, Murray Goldschmidt, Maor Dvir, Yitshak Hadani, Meir Hamo, Adir Cohen, Ilan Fischer, Aviva Rakover, and Guy Tamir, who read an earlier version of the article and made important and useful com- 


\section{S. S. RAKOVER}

ments on it.

\section{REFERENCES}

Baird, J. C., \& Noma, E. (1978). Fundamentals of scaling and psychophysics. New York: John Wiley.

Bayne, T. (2009). Consciousness. In J. Symons, \& P. Calvo (Eds.), The Rutledge companion to philosophy of psychology. London: Routledge.

Chalmers, D. (1996). The conscious mind: In search of a fundamental theory. New York: Oxford University Press.

Coombs, C. H., Dawes, R. M., \& Tversky, A. (1970). Mathematical psychology: An elementary introduction. Englewood Cliffs, NJ: Prentice Hall.

Cooper, R. (2004). Why Hacking is wrong about human kinds. British Journal for the Philosophy of Science, 55, 73-85. doi:10.1093/bjps $/ 55.1 .73$

Cooper, R., \& Shallice, T. (1995). SOAR and the ease for unified theories of cognition. Cognition, 55, 115-149. doi:10.1016/0010-0277(94)00644-Z

Cosmelli, D., Lachaux, J.-P., \& Thompson, E. (2007). Neurodynamical appoaches to consciousness. In P. Zelazo, M. Moscovitch, \& E. Thompson (Eds.), The Cambridge handbook of consciousness. Cambridge \& New York: Cambridge University Press.

De Houwer, J., Fiedler, K., \& Moors, A. (2011). Strengths and limitations of theoretical explanations in psychology: Introduction to the special section. Perspectives on Psychological Science, 6, 161-162. doi: $10.1177 / 1745691611400242$

Diener, E. (2010). Neuroimaging: Voodoo, new phrenology, or scientific breakthrough? Introduction to special section on fMRI. Perspectives on Psychological Science, 5, 714-715. doi:10.1177/1745691610388773

Garcia-Marques, L., \& Ferreira, M. B. (2011). Friends and foes of theory construction in psychological science: Vague dichotomies, unified theories of cognition, and the new experimentalism. Perspectives on Psychological Science, 6, 192-201. doi:10.1177/1745691611400239

Gescheider, G. A. (1997). Psychophysics: The fundamentals. Nahwah: LEA.

Gribbin, J. (2004). The scientists: A history of science told through the lives of its greatest inventors. New York: Random House.

Gross, R. (2009). Themes, issues and debates in psychology (3rd ed.). Abingdon: Hodder

Hacking, I. (1995). The looping effects of human kinds. In D. Sperber, D. Premack, \& A. J. Premack (Eds.), Causal cognition: A multi-disciplinary debate. Oxford: Clarendon Press.

Hand, D. J. (2004). Measurement theory and practice: The world through quantification. London: Arnold.

Hilgard, E. R., \& Bower, G. H. (1966). Theories of learning (3rd ed.). New York: Appleton-Century-Crofts.

Kim, J. (1996). Philosophy of mind. Boulder, CO: Westview Press.

Kimble, G. A. (1961). Higard and Marquis' conditioning and learning (2nd ed.). New York: Appleton-Century-Crofts.

Kouider, S. (2009). Neurobiological theories of consciousness. In W. Banks (Ed.), Encyclopedia of consciousness. Oxford: Elsevier Press. doi:10.1016/B978-012373873-8.00055-4

Kriegel, U. (2007). Philosophical theories of consciousness: Contemporary western perspectives. In P. Zelazo, M. Moscovitch, \& E. Thompson (Eds.), The Cambridge handbook of consciousness. Cambridge \& New York: Cambridge University Press.

Leahey, T. H. (1992). The mythical revolution of American psychology. American Psychologist, 47, 308-318. doi:10.1037/0003-066X.47.2.308

Leahey, T. H. (2004). A history of psychology (6th ed.). Upper Saddle River, NJ: Pearson/Prentice-Hall.

Levine, J. (1983). Materialism and qualia: The explanatory gap. Pacific Philosophical Quarterly, 64, 354-361.

Lewin, K. (1936). Principles of topological psychology. New York: McGraw-Hill. doi:10.1037/10019-000
Lewis, R. L. (2001). Cognitive theory, SOAR. In N. J. Smelser, \& P. B. Baltez (Eds.), International encyclopedia of the social and behavioral sciences. Amsterdam: Pergamon. doi:10.1016/B0-08-043076-7/01583-7

Ludwig, K. (2003). The mind-body problem: An overview. In S. P. Stich, \& T. A. Warfield (Eds.), The Blackwell guide to philosophy of mind (pp. 1-46). Malden, MA: Blackwell.

Marx, M. H., \& Cronan-Hillix, W. A. (1987). Systems and theories in psychology (4th ed.). New York: McGraw-Hill Book Company.

McCauley, R. N. (2007). Reduction: Models of cross-scientific relations and their implications for the psychology-neuroscience interface. In P. Thagard (Ed.), Philosophy of psychology and cognitive science. Amsterdam: Elsevier. doi:10.1016/B978-044451540-7/50021-9

McCauley, R. N., \& Bechtel, W. (2001). Explanatory pluralism and heuristic identity theory. Theory \& Psychology, 11, 736-760. doi:10.1177/0959354301116002

Macmillan, N. A., \& Creelman, C. D. (2005). Detection theory: A user's guide (2nd ed.). New York: Psychological Press.

Meiser, T. (2011). Much pain, little gain? Paradigm-specific models and methods in Experimental psychology. Perspectives on Psychological Science, 6, 183-191. doi:10.1177/1745691611400241

Michell, J. (1999). Measurement in psychology: Critical history of a methodological concept. Hillsdale, NJ: LEA. doi:10.1017/CBO9780511490040

Miller, G. A. (2010). Mistreating psychology in the decades of the brain. Perspectives on Psychological Science, 5, 716-743. doi:10.1177/1745691610388774

Neisser, U. (1967). Cognitive psychology. New York: Appleton-Century-Crofts.

Newell, A. (1973). You can't play 20 questions with nature and win. In W. G. Chase (Ed.), Visual information processing. San Diego, CA Academic Press.

Newell, A. (1992). SOAR as a unified theory of cognition: Issues and explanations. Behavioral and Brain Sciences, 15, 464-492. doi:10.1017/S0140525X00069740

Pachella, R. G. (1974). The interpretation of reaction time in information-processing research. In B. H. Kantowitz (Ed.), Human information processing: Tutorials in performance and cognition. LEA.

Palmer, S. E., \& Kimchi, R. (1986). The information processing approach to cognition. In T. J. Knapp, \& L. C. Robertson (Eds.), Approaches to cognition: Contrasts and controversies. Nahwah: LEA

Polger, T. (2004). Natural minds. Cambridge: MIT press.

Rakover, S. S. (1975). Tolerance of pain as a measure of fear. Learning and Motivation, 6, 43-61. doi:10.1016/0023-9690(75)90034-X

Rakover, S. S. (1980). Role of intertribal interval following escape or avoidance response in bar-press avoidance. Learning and Motivation, 11, 220-237. doi:10.1016/0023-9690(80)90014-4

Rakover, S. S. (1990). Metapsychology: Missing links in behavior, mind and science. New York: Paragon/Solomon.

Rakover, S. S. (1997). Can psychology provide a coherent account of human behavior? A proposed multiexplanation-model theory. Behavior and Philosophy, 25, 43-76.

Rakover, S. S. (2002a). Featural vs. configurational information in faces: A conceptual and empirical analysis. The British Journal of Psychology, 93, 1-30. doi:10.1348/000712602162427

Rakover, S. S. (2002b). Scientific rules of the game and the mind/body: A critique based on the theory of measurement. Journal of Consciousness Studies, 9, 52-58.

Rakover, S. S. (2007). To understand a cat: Methodology and philosophy. Amsterdam/Philadelphia: John Benjamins.

Richards, G. (2010). Putting psychology in its place: A critical historical overview (3rd ed.). London: Routledge.

Rowlands, M. (2009). The mind-body problem. In W. Banks (Ed.), Encyclopedia of Consciousness. Oxford: Elsevier Press. doi:10.1016/B978-012373873-8.00053-0

Schwartz, B. (1989). Psychology of learning and behavior. New York: Norton.

Shimamura, A. P. (2010). Bridging psychological and biological science: The good, and ugly. Perspectives on Psychological Science, 5, 


\section{S. S. RAKOVER}

772-775. doi: $10.1177 / 1745691610388781$

Stevens, S. S. (1975). Psychophysics: Introduction to its perceptual neural and social prospects. New York: John Wiley.

Sternberg, R. J. (2009). Cognitive psychology (5th ed.). Belmont, CA: Wadsworth.

Suppe, F. (1977). The structure of scientific theories (2nd ed.). Chicago, IL: University of Illinois Press.
Suppe, F. (1979). Theory structure. In P. D. Asquith, \& H. E. Kijburg Jr. (Eds.), Current research in philosophy of science. Michigan: Philosophy of Science Association.

Uttal, W. R. (2008). Time, space, and number in Physics and Psychology. Cornwall-on-Hudson, NY: Sloan Publishing.

Watson, J. B. (1913). Psychology as the behaviorist views it. Psychological Review, 20, 158-177. doi:10.1037/h0074428 